\title{
NEW STRUCTURE FOR ORTHOGONAL QUANTUM GROUP INVARIANTS
}

\author{
QINGTAO CHEN AND KEFENG LIU
}

(Communicated by Lei $\mathrm{Ni}$ )

\begin{abstract}
Based on the orthogonal Labastida-Mariño-Ooguri-Vafa conjecture made by L. Chen and Q. Chen (2012), we derive an infinite product formula for Chern-Simons partition functions, which generalizes Liu and Peng's recent results to the orthogonal case. Symmetry property of this new infinite product structure is also discussed.
\end{abstract}

\section{INTRODUCTION}

In 1984, Jones 9, 10 discovered a polynomial invariant of oriented knots. Later, using this result, the two-variable HOMFLY-PT [7,22] and one-variable Kauffman polynomials [11] of oriented and unoriented links were also discovered in 1985 and 1987 respectively. HOMFLY-PT polynomial generalizes both the Alexander and Jones polynomial. In 1990, the two-variable Kauffman polynomial [12] was also introduced, which only generalizes the Jones polynomial.

By using the Chern-Simons path integral method, Witten [29] gave a quantum field theory interpretation of the Jones polynomial. Witten [29] also predicted the existence of 3-manifold quantum invariants. Following this track, Reshetikhin and Turaev [26, 27] gave a construction of 3-manifold invariants by using quantum universal enveloping algebra (quantum group), $U_{q}\left(s l_{2}\right)$ at roots of unity, which led to the colored version of classical HOMFLY-PT and Kauffman polynomial invariants. Their work actually gave a unified understanding of the quantum group invariants of links. Here color means the representation of quantum groups. It turns out that a colored HOMFLY-PT invariant is a special linear quantum group invariant, i.e., the quantum group of $A_{n}$ type; while colored Kauffman invariant is a quantum group invariant of $B_{n}, C_{n}$ and $D_{n}$ type.

In a series of papers, Labastida, Mariño, Ooguri and Vafa [13, 14, 21 proposed a conjectural description of a deep relationship between reformulated invariants of colored HOMFLY-PT links in the early 2000s. This conjecture was proved by K. Liu and P. Peng in 17, 18.

In some sense the Labastida-Mariño-Ooguri-Vafa (LMOV) conjecture can be expressed purely by using mathematical language, i.e., irreducible representation of quantum groups. The physics background of this conjecture can be dated back to 't Hooft's seminal work on large $N$ expansion of $U(N)$ gauge field theories in 1974. Gopakumar and Vafa 8] described the exact theory that closed topological

Received by the editors October 12, 2013.

2010 Mathematics Subject Classification. Primary 57M27; Secondary 81R50. 
string theory on the resolved conifold is dual to the $U(N)$ Chern-Simons theory on $S^{3}$. The Gromov-Witten theory of the resolved conifold actually corresponds to the Chern-Simons theory of an unknot. The LMOV conjecture considers the more general case when the link or knot is nontrivial and the corresponding Wilson loop expectation values, i.e., a colored HOMFLY-PT polynomial of the link. So the LMOV conjecture could be viewed as a counterpart of the invariant GopakumarVafa conjecture.

Previously, people thought that only HOMFLY-PT polynomial could be expressed as a series in $q-q^{-1}$ and $t^{ \pm 1}$ with integer coefficients; while the colored HOMFLY-PT invariant was just a Laurent polynomial of $q^{ \pm 1}$ and $t^{ \pm 1}$ with rational coefficients. The LMOV conjecture predicts an intrinsic symmetry of $q-q^{-1}$ about reformulated invariants of the colored HOMFLY-PT invariant and hidden integrality encoded in the colored HOMFLY-PT invariant.

In 2009, the orthogonal LMOV conjecture was also formulated by Lin Chen and Qingtao Chen [4 6]. and Marcos Mariño 20]. Chen and Chen's formulation put an emphasis solely on the colored Kauffman; while Mariño's put an emphasis on the relation between the composite colored HOMFLY-PT invariants and the colored Kauffman invariants.

More recently, Kefeng Liu and Pan Peng [19] obtained a new structure of the colored HOMFLY-PT polynomial; namely, that the Chern-Simons partition function appearing in the original LMOV conjecture can be expressed as an infinite product, which indicates some potential modularity of the Chern-Simons partition function [19].

In this paper, an infinite product expression for the orthogonal Chern-Simons partition function appearing in the orthogonal LMOV type conjecture [4-6] is established and the case of an unknot is presented in an explicit formula.

This paper is organized as follows. In Section 2, we introduce the basic setups for the quantum group invariant of links. In Section 3, we describe the original and the orthogonal LMOV conjecture as well as notation. In Section 4, we derive the orthogonal Chern-Simons partition function as an infinite product and illustrate an example of the unknot. In Section 5, we discuss the symmetric properties associated to this infinite product structure.

\section{QUANTUM INVARIANTS OF LINKS}

Let $\mathfrak{g}$ be a finite dimensional complex semi-simple Lie algebra of rank $N$ with Cartan matrix $\left(C_{i j}\right)$. Let $U_{q}(\mathfrak{g})$ be the quantum enveloping algebra of $\mathfrak{g}$. Let $V$ be a vector space over a field $k$. A linear automorphism $c$ of $V \otimes V$ is said to be an $R$-matrix if it is a solution of the following Yang-Baxter equation:

$$
\left(c \otimes i d_{V}\right)\left(i d_{V} \otimes c\right)\left(c \otimes i d_{V}\right)=\left(i d_{V} \otimes c\right)\left(c \otimes i d_{V}\right)\left(i d_{V} \otimes c\right)
$$

that holds in the automorphism group of $V \otimes V \otimes V$.

It is well known that the solution of the Yang-Baxter equation provides a representation of the braid group. The solution we used is the following so-called universal $R$-matrix:

$$
R=q^{\sum_{i, j} C_{i j}^{-1} H_{i} \otimes H_{j}} \prod_{\beta} \exp _{q}\left[\left(1-q^{-2}\right) X_{\beta}^{+} \otimes X_{\beta}^{-}\right],
$$


where $\beta$ runs over positive roots of $\operatorname{sl}(N, \mathbf{C}),\left(C_{i j}\right)$ is the Cartan matrix, and $q$-exponential is given by

$$
\exp _{q}[x]=\sum_{k=0}^{\infty} q^{\frac{1}{2} k(k+1)} \frac{x^{k}}{[k]_{q} !}
$$

where

and $[n]=q^{n}-q^{-n}$.

$$
[k]_{q} !=[k]_{q} \cdot[k-1]_{q} \cdots[1]_{q},[k]_{q}=\frac{[k]}{[1]}
$$

Given a link $\mathcal{L}$ with $L$ components, it is well known that $\mathcal{L}$ can be represented by an element in some braid group $B_{m}$ with $m$ strands. For each component, we associate to it an irreducible representation $A^{\alpha}$ of quantized universal enveloping algebra $U_{q}(\operatorname{sl}(N, \mathbb{C})) . A^{\alpha}$ is labeled by the highest weight $\Lambda_{\alpha}$. As usual, we associate them with the Young diagrams. Without loss of generality, one can assume the first $m_{1}$ strands correspond to the first component, the second $m_{2}$ strands correspond to the second component, and so on. Let

$$
\widehat{V}=\bigotimes_{\alpha=1}^{L} V_{\Lambda_{\alpha}}^{m_{\alpha}}
$$

and write the braiding $\check{R}=P_{12} R: V \otimes W \rightarrow W \otimes V$, where $P_{12}(s \otimes t)=t \otimes s$.

For a generator of the braid group $B_{m}, \sigma_{i}$, define

$$
\pi\left(\sigma_{i}^{ \pm 1}\right)=I d_{V_{1}} \otimes I d_{V_{2}} \otimes \cdots \otimes I d_{V_{i-1}} \otimes \check{R}^{ \pm 1} \otimes \cdots \otimes I d_{V_{m}} .
$$

The quantum group invariants of the link $\mathcal{L}$ is defined as follows:

$$
W_{A^{1}, \cdots, A^{L}}^{\mathfrak{g}}(\mathcal{L})=q^{d(\mathcal{L})} \operatorname{Tr}_{\widehat{V}}\left(\mu^{m} \cdot \pi(\mathcal{L})\right),
$$

where $\mu=q^{\rho^{*}}, \rho^{*}$ is the element in $\mathfrak{h} \subset U_{q}(\mathfrak{h})$ corresponding to the Weyl vector (i.e. the sum of fundamental weights) under the natural isomorphism $\mathfrak{h} \simeq \mathfrak{h}^{*}$ and $d(\mathcal{L})$ and is given by the following formula:

$$
d(\mathcal{L})=-\sum_{\alpha=1}^{L} \omega\left(\mathcal{K}_{\alpha}\right)\left(\Lambda_{\alpha}, \Lambda_{\alpha}+2 \rho\right)+\frac{2}{N} \sum_{\alpha<\beta}^{L} l k\left(\mathcal{K}_{\alpha}, \mathcal{K}_{\beta}\right) l_{\alpha} l_{\beta}
$$

Special case 1 . For the unknot $\bigcirc, W_{A}(\bigcirc)$ is the quantum $\operatorname{dimension} \operatorname{dim}_{q}\left(V_{A}\right)$ of the corresponding representation space $V_{A}$.

Special case 2. If $\mathfrak{g}=s l_{N}$ and $A^{1}=A^{2}=\cdots=A^{L}=(1)$, the quantum group invariant of links is equal to the HOMFLY polynomial at $t=q^{N}$ up to a universal factor $\frac{t-t^{-1}}{q-q^{-1}}$.

Special case 3 . If $\mathfrak{g}=s o_{2 N+1}$ and $A^{1}=A^{2}=\cdots=A^{L}=(1)$, the quantum group invariant of links is equal to the Kauffman polynomial at $t=q^{2 N}$ up to a universal factor $1+\frac{t-t^{-1}}{q-q^{-1}}$ and some $t$ power of the linking numbers.

Thus the quantum group invariants associated to $\mathfrak{g}=s l_{N}$ and $\mathfrak{g}=s o_{2 N+1}$ are called the colored HOMFLY invariants and the colored Kauffman invariants respectively.

Actually the irreducible representation of the quantum groups of special linear and orthogonal cases can be labeled by the Young Tableau. Now we would like 
to introduce some basic notation of the partition and the corresponding Young Tableau.

A partition of $n$ is a tuple of positive integers $\mu=\left(\mu_{1}, \mu_{2}, \ldots, \mu_{k}\right)$ such that $|\mu| \triangleq \sum_{i=1}^{k} \mu_{i}=n$ and $\mu_{1} \geq \mu_{2} \geq \cdots \geq \mu_{k}>0$, where $|\mu|$ is called the degree of $\mu$ and $k$ is called the length of $\mu$, denoted by $\ell(\mu)$. A partition can be represented by a Young diagram, for example, partition $(5,4,2,1)$ can be identified as the following Young diagram.

Denote by $\mathcal{P}$ the set of all Young diagrams. Let $\chi_{A}$ be the character of irreducible representation of symmetric group, labeled by partition $A$. Given a partition $\mu$, define $m_{j}=\#\left(\mu_{k}=j ; k \geq 1\right)$. The order of the conjugate class of type $\mu$ is given by:

$$
\mathfrak{z}_{\mu}=\prod_{j \geq 1} j^{m_{j}} m_{j} !
$$

The theory of symmetric functions has a close relationship with the representations of a symmetric group. The symmetric power functions of a given set of variables $x=\left\{x_{j}\right\}_{j \geq 1}$ are defined as the direct limit of the Newton polynomials:

$$
p_{n}(x)=\sum_{j=1}^{\infty} x_{j}^{n}, \quad p_{\mu}(x)=\prod_{i=1}^{\ell(\mu)} p_{\mu_{i}}(x) .
$$

We will consistently denote by $\mathcal{L}$ a link and by $L$ the number of components in $\mathcal{L}$.

The irreducible $U_{q}(\mathfrak{g})$ modules associated to $\mathcal{L}$ will be labeled by their highest weights, thus by Young diagrams. We usually denote it by a vector form $\vec{A}=$ $\left(A^{1}, \ldots, A^{L}\right)$.

Let $\vec{x}=\left(x^{1}, \ldots, x^{L}\right)$ be $L$ sets of variables, each of which is associated to a component of $\mathcal{L}$, and $\vec{\mu}=\left(\mu^{1}, \ldots, \mu^{L}\right) \in \mathcal{P}^{L}$ be a tuple of $L$ partitions. Define:

$$
\begin{array}{lll}
{[\mu]=\prod_{i=1}^{\ell(\mu)}\left[\mu_{i}\right],} & {[\vec{\mu}]=\prod_{\alpha=1}^{L}\left[\mu^{\alpha}\right],} & \mathfrak{z}_{\vec{\mu}}=\prod_{\alpha=1}^{L} \mathfrak{z}_{\mu^{\alpha}}, \\
\chi_{\vec{A}}\left(C_{\vec{\mu}}\right)=\prod_{\alpha=1}^{L} \chi_{A^{\alpha}}\left(C_{\mu^{\alpha}}\right), & s_{\vec{A}}(\vec{x})=\prod_{\alpha=1}^{L} s_{A^{\alpha}}\left(x^{\alpha}\right), & p_{\vec{\mu}}(\vec{x})=\prod_{\alpha=1}^{L} p_{\mu^{\alpha}}\left(x^{\alpha}\right) .
\end{array}
$$

When we consider the orthogonal quantum group invariants, we need to study the Brauer algebra $B r_{n}$ which contains the group algebra $\mathbb{C}\left[S_{n}\right]$ as a direct summand. Thus all the irreducible representations of $S_{n}$ are also irreducible representations of $B r_{n}$, labeled by partitions of the integer $n$. Indeed, the set of irreducible representations of $B r_{n}$ are bijective to the set of partitions of the integers $n-2 k$, where $k=0,1, \cdots,\left[\frac{n}{2}\right][24,28]$. Thus the semi-simple algebra $B r_{n}$ can be decomposed into the direct sum of simple algebras

$$
B r_{n} \cong \bigoplus_{k=0}^{\left[\frac{n}{2}\right]} \bigoplus_{\lambda \vdash n-2 k} M_{d_{\lambda} \times d_{\lambda}}(\mathbb{C}) .
$$

The work of Beliakova and Blanchet [1] constructed an explicit basis of the above decomposition. An up and down tableau $\Lambda=\left(\lambda_{1}, \lambda_{2}, \cdots, \lambda_{n}\right)$ is a tube of $n$ Young diagrams such that $\lambda_{1}=(1)$ and each $\lambda_{i}$ is obtained by adding or removing one 
box from $\lambda_{i-1}$. Let $\lambda$ be a partition of $n-2 k$. Denote by $|\Lambda|=\lambda$ if $\lambda_{n}=\lambda$, and we say an up and down tableau $\Lambda$ is of shape $\lambda$. There is a minimal path idempotent $p_{\Lambda} \in B r_{n}$ associated to each $\Lambda$. Then the minimal central idempotent $\pi_{\lambda}$ of $B r_{n}$ corresponding to the irreducible representation labeled by $\lambda$ is given by

$$
\pi_{\lambda}=\sum_{|\Lambda|=\lambda} p_{\Lambda}
$$

In particular, the dimension of the irreducible representations $d_{\lambda}$ is the number of up and down tableau of shape $\lambda$. More details can be found in [1,28,

The table of the characters and orthogonal relations can be found in [23 25]. The values of a character of $B r_{n}$ is completely determined by its values on the set of elements $e^{k} \otimes \gamma_{\lambda}$, where $e$ is the conjugacy class of $e_{1}, \cdots, e_{n-1}$ and $\gamma_{\lambda}$ is the conjugacy class in $S_{n-2 k}$ labeled by the partition $\lambda$ of $n-2 k$. The notion $e^{k} \otimes \gamma_{\lambda}$ stands for the tangle in the following diagram:

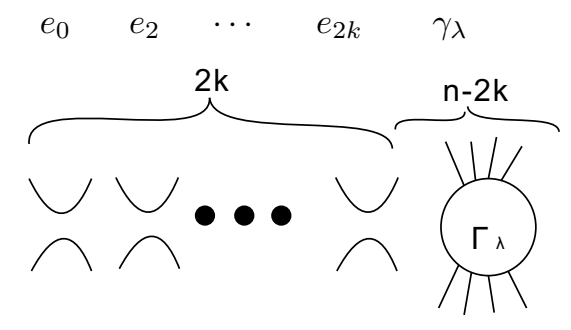

where $\Gamma_{\lambda}$ is a diagram in the conjugacy class of $S_{n-2 k}$ labeled by a partition $\lambda$ of $n-2 k$.

Denote by $\chi_{A}$ the character of the irreducible representation of $B r_{n}$ labeled by a partition $A \vdash n-2 k$ for some $k$, and denote by $\chi_{B}^{S_{n}}$ the character of the irreducible representation of $S_{n}$ labeled by a partition $B \vdash n$. It is known that when $A$ is a partition of $n$, then $\chi_{A}\left(e^{m} \otimes \gamma_{\lambda}\right)=0$ for all $m>0$ and partition $\lambda \vdash n-2 m$, and $\chi_{A}\left(\gamma_{\mu}\right)=\chi_{A}^{S_{n}}\left(\gamma_{\mu}\right)$ for partition $\mu \vdash n$ coincide with the characters of the permutation group $S_{n}[24$.

\section{Labastida-Mariño-Ooguri-VAFA tYPe CONJECTURE FOR THE COLORED KAUFFMAN INVARIANT}

Let's quickly review the original LMOV conjecture first.

For each link $\mathcal{L}$, the type- $A$ Chern-Simons partition function of $\mathcal{L}$ is defined by

$$
Z_{C S}^{S L}(\mathcal{L} ; q, t ; \vec{x})=\sum_{\vec{A} \in \mathcal{P}^{L}} W_{\vec{A}}^{S L}(\mathcal{L} ; q, t) s_{\vec{A}}(\vec{x})=1+\sum_{\vec{\mu} \neq \overrightarrow{0}} Z_{\vec{\mu}}^{S L} p_{\vec{\mu}}(\vec{x}),
$$

where $s_{\vec{A}}(\vec{x})$ are the Schur polynomials.

The original LMOV conjecture describes a very subtle structure of $Z_{C S}^{S L}(\mathcal{L} ; q, t ; \vec{x})$, which was proved by Kefeng Liu and Pan Peng [17, 18, based on the cabling technique and a careful degree analysis of the cut-join equation. As an application, the LMOV conjecture gives highly nontrivial relations between colored HOMFLY invariants. The first such relation is the classical Lickorish-Millett theorem [15.

The study of the colored Kauffman invariants is more difficult. For instance, the definition of the Chern-Simons partition function for the orthogonal quantum groups involves the representations of Brauer centralizer algebras, which admit a 
more complicated orthogonal relation [23 25]. In a joint work with Lin Chen, we [5] rigorously formulate the orthogonal quantum group version of LMOV conjecture by using the representation of the Brauer centralizer algebra.

Now we set $\mathfrak{g}=\mathrm{sO}_{2 N+1}$.

Let $\mathcal{L}$ be a link with $L$ components and

$$
p b_{n}(z)=\sum_{j=-\infty}^{\infty} z_{j}^{n}, \quad p b_{\mu}(z)=\prod_{i=1}^{\ell(\mu)} p b_{\mu_{i}}(z), \quad p b_{\vec{\mu}}(\vec{z})=\prod_{\alpha=1}^{L} p b_{\mu^{\alpha}}\left(z^{\alpha}\right) .
$$

Let $Z_{C S}^{S O}(\mathcal{L}, q, t)$ be the orthogonal Chern-Simons partition function defined by

$$
Z_{C S}^{S O}(\mathcal{L} ; q, t ; \vec{z})=\sum_{\vec{\mu} \in \mathcal{P}^{L}} \frac{p b_{\vec{\mu}}(\vec{z})}{\mathfrak{z}_{\vec{\mu}}} \sum_{\vec{A} \in \widehat{B r}_{|\vec{\mu}|}} \chi_{\vec{A}}\left(\gamma_{\vec{\mu}}\right) W_{\vec{A}}^{S O}(\mathcal{L} ; q, t),
$$

where $\mathfrak{z} \vec{\mu}=\frac{\|\vec{\mu}\| !}{\left|C_{\vec{\mu}}\right|},|\vec{\mu}|=\left(d^{1}, \ldots, d^{L}\right), \widehat{B r}_{|\vec{\mu}|}$ denotes the set $\widehat{B r}_{d^{1}} \times \cdots \times \widehat{B r}_{d^{L}}$ (every element is a representation of the Brauer algebra), $\vec{\mu}=\left(\mu^{1}, \ldots, \mu^{L}\right)$ for partitions $\mu^{i}$ of $d^{i} \in \mathbb{Z}$ and $\chi_{\vec{A}}\left(\gamma_{\vec{\mu}}\right)=\prod_{i=1}^{L} \chi_{A^{i}}\left(\gamma_{\mu^{i}}\right)$ for the character $\chi_{A^{i}}$ of $B r_{d^{i}}$ labeled by $A^{i}$.

Expend the free energy

$$
F_{C S}^{S O}(\mathcal{L} ; q, t ; \vec{z})=\log Z_{C S}^{S O}(\mathcal{L} ; q, t ; \vec{z})=\sum_{\vec{\mu} \neq \overrightarrow{0}} F_{\vec{\mu}}^{S O}(\mathcal{L} ; q, t) p b_{\vec{\mu}}(\vec{z}) .
$$

Then the reformulated invariants are defined by

$$
g_{\vec{\mu}}(\mathcal{L} ; q, t)=\sum_{k \mid \vec{\mu}} \frac{\mu(k)}{k} F_{\vec{\mu} / k}\left(\mathcal{L} ; q^{k}, t^{k}\right) .
$$

The orthogonal LMOV conjecture was formulated by L. Chen and Q. Chen [4 6] as follows.

Conjecture 3.1 (Orthorgonal LMOV, Chen-Chen [4-6]). Let

$$
\frac{\mathfrak{z}_{\vec{\mu}}[1]^{2} \cdot\left[g_{\vec{\mu}}(\mathcal{L} ; q, t)-g_{\vec{\mu}}(\mathcal{L} ; q,-t)\right]}{2[\vec{\mu}]}=\sum_{g=0}^{\infty} \sum_{\beta \in \mathbb{Z}} N_{\vec{\mu}, g, \beta}\left(q-q^{-1}\right)^{g} t^{\beta},
$$

where $N_{\vec{\mu}, g, \beta}$ are the integer coefficients and vanish for sufficiently large $g$ and $|\beta|$.

This conjecture is a rigorous mathematical formulation of the LMOV type conjecture about the colored Kauffman invariant; while in [2, 20, their conjecture emphasizes the relationship between colored HOMFLY-PT and colored Kauffman invariants. The integer coefficients $N_{\vec{\mu}, g, \beta}$ are closely related to the BPS numbers.

\section{Infinite PRODUCT Formula For orthogonal Chern-Simons PARTITION FUNCTIONS}

To derive an infinite product formula, we will state the result for a knot first, since the notation in the computation for a knot is relatively simpler. 
4.1. The case of a knot. Given $z=\left\{z_{i}\right\}_{-\infty<i<\infty}, x=\left\{x_{j}\right\}_{j \geq 1}$, define

$$
x * y=\left\{x_{i} \cdot y_{j}\right\}_{-\infty<i<\infty, j \geq 1} .
$$

We also define $z^{d}=\left\{z_{i}^{d}\right\}_{-\infty<i<\infty}$. The $d$-th Adam operation of a type-B Schur function is given by $s b_{A}\left(z^{d}\right)$.

Introducing variables $q^{\rho}=\left\{-q^{2 j-1}\right\}_{j \geq 1}$, we have

$$
p_{n}\left(q^{\rho}\right)=\frac{1}{[n]},
$$

where we assume $|q|<1$.

Set $w=z * q^{\rho}$. Then we have

$$
p b_{n}(w)=\frac{p b_{n}(z)}{[n]}
$$

and

$$
p b_{\mu}(w)=\frac{p b_{\mu}(z)}{[\mu]} .
$$

Consider the free energy weighted by the $p b_{\mu}(w)$. The orthogonal LMOV conjecture implies the following reformulation of the free energy:

$$
F^{S O}(\mathcal{K} ; q, t ; w)=\sum_{d=1}^{\infty} \sum_{\mu \neq 0} \frac{1}{d} g_{\mu}\left(\mathcal{K} ; q^{d}, t^{d}\right) p b_{\mu}\left(w^{d}\right)
$$

and

$$
g_{\mu}(\mathcal{K} ; q, t)-g_{\mu}(\mathcal{K} ; q,-t)=\frac{2[\mu]}{\mathfrak{z}_{\mu}[1]^{2}} \sum_{g=0}^{\infty} \sum_{\beta \in \mathbb{Z}} N_{\vec{\mu}, g, \beta}\left(q-q^{-1}\right)^{g} t^{\beta}
$$

There exists integers $n_{B ; g, \beta}$ such that

$$
\sum_{g=0}^{\infty} N_{B, g, \beta}\left(q-q^{-1}\right)^{g}=\sum_{g=0}^{\infty} n_{B, g, \beta} \sum_{k=0}^{g} q^{g-2 k}
$$

By the orthogonal LMOV conjecture, $N_{B ; g, \beta}$ vanish for sufficiently large $g$ and $|\beta|$, thus $n_{B ; g, \beta}$ vanish for sufficiently large $g$ and $|\beta|$. 
We have

$$
\begin{aligned}
& F^{S O}(\mathcal{K} ; q, t ; w)-F^{S O}(\mathcal{K} ; q,-t ; w) \\
= & \sum_{d=1}^{\infty} \sum_{\mu \neq 0} \frac{1}{d} g_{\mu}\left(\mathcal{K} ; q^{d}, t^{d}\right) p b_{\mu}\left(w^{d}\right)-\sum_{d=1}^{\infty} \sum_{\mu \neq 0} \frac{1}{d} g_{\mu}\left(\mathcal{K} ; q^{d},(-1)^{d} t^{d}\right) p b_{\mu}\left(w^{d}\right) \\
= & \sum_{d \in O \mathbb{Z}_{+}} \sum_{\mu \neq 0} \frac{1}{d}\left(g_{\mu}\left(\mathcal{K} ; q^{d}, t^{d}\right)-g_{\mu}\left(\mathcal{K} ; q^{d},-t^{d}\right)\right) p b_{d \mu}(w) \\
= & \sum_{d \in O \mathbb{Z}_{+}} \sum_{\mu \neq 0} \frac{1}{d} \frac{2[d \mu]}{\mathfrak{z}_{\mu}[d]^{2}} \sum_{g=0}^{\infty} \sum_{\beta \in \mathbb{Z}} N_{\mu, g, \beta}\left(q^{d}-q^{-d}\right)^{g} t^{d \beta} p b_{d \mu}(w) \\
= & \sum_{d \in O \mathbb{Z}_{+}} \sum_{\mu \neq 0} \frac{1}{d} \frac{2}{\mathfrak{z}_{\mu}[d]^{2}} \sum_{g=0}^{\infty} \sum_{\beta \in \mathbb{Z}} N_{\mu, g, \beta}\left(q^{d}-q^{-d}\right)^{g} t^{d \beta} p b_{d \mu}(z) \\
= & \sum_{d \in O \mathbb{Z}_{+}} \sum_{\mu \neq 0} \frac{1}{d} \frac{2}{\mathfrak{z}_{\mu}[d]^{2}} \sum_{g=0}^{\infty} \sum_{\beta \in \mathbb{Z} k=0}^{g} \sum_{\mu, g, \beta} q^{(g-2 k) d} t^{d \beta} p b_{d \mu}(z) \\
= & \sum_{d \in O \mathbb{Z}_{+}} \sum_{\mu \neq 0} \frac{1}{d} \frac{2}{\mathfrak{z}_{\mu}} \sum_{m=1}^{\infty} m q^{2 m d} \sum_{g=0}^{\infty} \sum_{\beta \in \mathbb{Z} k=0}^{g} \sum_{\mu, g, \beta} q^{(g-2 k) d} t^{d \beta} p b_{d \mu}(z) \\
= & \sum_{d \in O \mathbb{Z}_{+}} \sum_{\mu \neq 0} \frac{1}{d} \frac{2}{\mathfrak{z}_{\mu}} \sum_{g=0}^{\infty} \sum_{\beta \in \mathbb{Z} m=1}^{\infty} \sum_{g}^{\infty} \sum_{k=0}^{\infty} m n_{\mu, g, \beta} q^{(g-2 k+2 m) d} t^{d \beta} p b_{d \mu}(z) \\
= & \sum_{\mu \neq 0}^{\infty} \sum_{g=0}^{\infty} \sum_{\beta \in \mathbb{Z} m=1}^{\infty} \sum_{m} \sum_{k=0}^{m} \frac{m n_{\mu, g, \beta}}{\mathfrak{z}_{\mu}} \sum_{d \in O \mathbb{Z}_{+}} \frac{2}{d} q^{(g-2 k+2 m) d} t^{d \beta} p b_{d \mu}(z),
\end{aligned}
$$

where $O \mathbb{Z}_{+}=\{1,3,5, \ldots\}$ denotes the set of all positive odd integers.

Now we analyze the following computation in detail:

$$
\begin{aligned}
& \sum_{d \in O \mathbb{Z}_{+}} \frac{2}{d} q^{(g-2 k+2 m) d} t^{d \beta} p b_{d \mu}(z) \\
= & \sum_{d \in O \mathbb{Z}_{+}} \frac{2}{d} q^{(g-2 k+2 m) d} t^{d \beta} \prod_{j=1}^{\ell(\mu)}\left(\sum_{i=-\infty}^{\infty}\left(z_{i}\right)^{d \mu_{j}}\right) \\
= & \sum_{d \in O \mathbb{Z}_{+}} \frac{2}{d} q^{(g-2 k+2 m) d} t^{d \beta} \sum_{i_{1}, \ldots, i_{\ell(\mu)}}\left(z_{i_{1}}^{\mu_{1}} z_{i_{2}}^{\mu_{2}} \cdots z_{i_{\ell(\mu)}}^{\mu_{\ell(\mu)}}\right)^{d} \\
= & \sum_{i_{1}, \ldots, i_{\ell(\mu)}} \sum_{d \in O \mathbb{Z}_{+}} \frac{2}{d}\left(q^{g-2 k+2 m} t^{\beta} z_{i_{1}}^{\mu_{1}} z_{i_{2}}^{\mu_{2}} \cdots z_{i_{\ell(\mu)}}^{\mu_{\ell(\mu)}}\right)^{d} .
\end{aligned}
$$


Now we compute the series $\sum_{d \in O \mathbb{Z}_{+}} \frac{2}{d} x^{d}$ as follows:

$$
\begin{aligned}
& \sum_{d \in O \mathbb{Z}_{+}} \frac{2}{d} x^{d} \\
& =2\left(\sum_{d=1}^{\infty} \frac{1}{d} x^{d}-\sum_{d \in 2 \mathbb{Z}_{+}} \frac{1}{d} x^{d}\right) \\
& =2\left(\sum_{d=1}^{\infty} \frac{1}{d} x^{d}-\sum_{d=1}^{\infty} \frac{1}{2 d} x^{2 d}\right) \\
& =2\left(-\log (1-x)+\frac{1}{2} \log \left(1-x^{2}\right)\right) \\
& =\log \frac{1+x}{1-x}
\end{aligned}
$$

where $2 \mathbb{Z}_{+}=\{2,4,6, \ldots\}$ denotes the set of all positive even integers.

Thus we obtain

$$
\begin{aligned}
& F^{S O}(\mathcal{K} ; q, t ; w)-F^{S O}(\mathcal{K} ; q,-t ; w) \\
& =\sum_{\mu \neq 0} \sum_{g=0}^{\infty} \sum_{\beta \in \mathbb{Z}} \sum_{m=1}^{\infty} \sum_{k=0}^{g} \frac{m n_{\mu, g, \beta}}{\mathfrak{z}_{\mu}} \sum_{d \in O \mathbb{Z}_{+}} \frac{2}{d} q^{(g-2 k+2 m) d} t^{d \beta} p b_{d \mu}(z) \\
& =\sum_{\mu \neq 0} \sum_{g=0}^{\infty} \sum_{\beta \in \mathbb{Z} m=1}^{\infty} \sum_{k=0}^{g} \frac{m n_{\mu, g, \beta}}{\mathfrak{z}_{\mu}} \sum_{i_{1}, \ldots, i_{l(\mu)}} \sum_{d \in O \mathbb{Z}_{+}} \frac{2}{d}\left(q^{g-2 k+2 m} t^{\beta} z_{i_{1}}^{\mu_{1}} z_{i_{2}}^{\mu_{2}} \cdots z_{i_{\ell(\mu)}}^{\mu_{\ell(\mu)}}\right)^{d} \\
& =\sum_{\mu \neq 0} \sum_{g=0}^{\infty} \sum_{\beta \in \mathbb{Z}} \sum_{m=1}^{\infty} \sum_{k=0}^{g} \frac{m n_{\mu, g, \beta}}{\mathfrak{z}_{\mu}} \sum_{i_{1}, \ldots, i_{l(\mu)}} \log \frac{1+q^{g-2 k+2 m} t^{\beta} z_{i_{1}}^{\mu_{1}} z_{i_{2}}^{\mu_{2}} \cdots z_{i_{\ell(\mu)}}^{\mu_{\ell(\mu)}}}{1-q^{g-2 k+2 m} t^{\beta} z_{i_{1}}^{\mu_{1}} z_{i_{2}}^{\mu_{2}} \cdots z_{i_{\ell(\mu)}}^{\mu_{\ell(\mu)}}} \\
& =\sum_{\mu \neq 0} \sum_{g=0}^{\infty} \sum_{\beta \in \mathbb{Z} m=1}^{\infty} \sum_{k=0}^{g} \frac{m n_{\mu, g, \beta}}{\mathfrak{z}_{\mu}} \log \prod_{i_{1}, \ldots, i_{l(\mu)}} \frac{1+q^{g-2 k+2 m} t^{\beta} z_{i_{1}}^{\mu_{1}} z_{i_{2}}^{\mu_{2}} \cdots z_{i_{\ell(\mu)}}^{\mu_{\ell(\mu)}}}{1-q^{g-2 k+2 m} t^{\beta} z_{i_{1}}^{\mu_{1}} z_{i_{2}}^{\mu_{2}} \cdots z_{i_{\ell(\mu)}}^{\mu_{\ell(\mu)}}} .
\end{aligned}
$$

Define the symmetric product as shown in the following formula:

$$
\left\langle 1 \pm \omega z^{\mu}\right\rangle=\prod_{i_{1}, \ldots, i_{\ell(\mu)}}\left(1 \pm \omega z_{i_{1}}^{\mu_{1}} \cdots z_{i_{\ell(\mu)}}^{\mu_{\ell(\mu)}}\right) .
$$

Thus we have

$$
\begin{aligned}
& F^{S O}(\mathcal{K} ; q, t ; w)-F^{S O}(\mathcal{K} ; q,-t ; w) \\
& =\sum_{\mu \neq 0} \sum_{g=0}^{\infty} \sum_{\beta \in \mathbb{Z} m=1} \sum_{k=0}^{\infty} \sum_{k}^{g} \frac{m n_{\mu, g, \beta}}{\mathfrak{z}_{\mu}} \log \frac{\left\langle 1+q^{g-2 k+2 m} t^{\beta} z^{\mu}\right\rangle}{\left\langle 1-q^{g-2 k+2 m} t^{\beta} z^{\mu}\right\rangle} \\
& =\log \prod_{\mu \neq 0} \prod_{g=0}^{\infty} \prod_{\beta \in \mathbb{Z} m=1}^{\infty} \prod_{m=0}^{g}\left(\frac{\left\langle 1+q^{g-2 k+2 m} t^{\beta} z^{\mu}\right\rangle}{\left\langle 1-q^{g-2 k+2 m} t^{\beta} z^{\mu}\right\rangle}\right)^{\frac{m n_{\mu, g, \beta}}{\mathfrak{z}}} .
\end{aligned}
$$

Now we obtain the infinite product formula for the orthogonal Chern-Simons partition function of knots. 
Theorem 4.1 (Orthogonal infinite product formula for knots). Based on Conjecture 3.1, the Chern-Simons partition function for orthogonal quantum group invariants can be expressed as the following infinite product formula:

$$
\frac{Z_{C S}^{S O}(\mathcal{K} ; q, t ; w)}{Z_{C S}^{S O}(\mathcal{K} ; q,-t ; w)}=\prod_{\mu \neq 0} \prod_{g=0}^{\infty} \prod_{\beta \in \mathbb{Z} m=1} \prod_{k=0}^{g} \prod_{k=0}^{g}\left(\frac{\left\langle 1+q^{g-2 k+2 m} t^{\beta} z^{\mu}\right\rangle}{\left\langle 1-q^{g-2 k+2 m} t^{\beta} z^{\mu}\right\rangle}\right)^{\frac{m_{n, g, \beta}}{{ }^{\prime \mu}}}
$$

4.2. The case of a link. Now we consider the case of a link.

Given a link $\mathcal{L}$ of $L$ components, let $\vec{w}=\left(w^{1}, \ldots, w^{L}\right)$ and $\vec{z}=\left(z^{1}, \ldots, z^{L}\right)$ satisfying $w^{i}=z^{i} * q^{\rho}$, for $i=1, \ldots, L$.

We generalize the symmetric product to the case of a link as follows:

$$
\begin{aligned}
& \left\langle 1 \pm \omega\left(z^{1}\right)^{\mu^{1}} \cdots\left(z^{L}\right)^{\mu^{L}}\right\rangle \\
& \left.=\prod_{\left.\left.i_{1,1}, \ldots, i_{1, \ell(\mu}\right), \ldots, i_{L, 1}, \ldots, i_{L, \ell(\mu}\right)}\left(1 \pm \omega \prod_{\alpha=1}^{L}\left(\left(z_{i_{\alpha, 1}}^{\alpha}\right)^{\mu_{1}^{\alpha} \cdots\left(z_{i_{\alpha, \ell}\left(\mu^{\alpha}\right)}^{\alpha}\right.}\right)^{\left.\mu_{\ell\left(\mu^{\alpha}\right)}^{\alpha}\right)}\right)\right) .
\end{aligned}
$$

There exist integers $n_{\vec{B} ; g, \beta}$ such that

$$
\sum_{g=0}^{\infty} N_{\vec{B}, g, \beta}\left(q-q^{-1}\right)^{g}=\sum_{g=0}^{\infty} n_{\vec{B}, g, \beta} \sum_{k=0}^{g} q^{g-2 k} .
$$

In a similar way, the infinite product formula for the orthogonal Chern-Simons partition function of a link can be obtained as follows.

Theorem 4.2 (Orthogonal infinite product formula for links). Based on Conjecture 3.1, the Chern-Simons partition function for orthogonal quantum group invariants can be expressed as the following infinite product formula:

$$
\begin{aligned}
& \frac{Z_{C S}^{S O}(\mathcal{L} ; q, t ; \vec{w})}{Z_{C S}^{S O}(\mathcal{L} ; q,-t ; \vec{w})} \\
& =\prod_{\vec{\mu} \neq \overrightarrow{0}} \prod_{g=0}^{\infty} \prod_{\beta \in \mathbb{Z}} \prod_{m=1}^{\infty} \prod_{k=0}^{g}\left(\frac{\left\langle 1+q^{g-2 k+2 m} t^{\beta}\left(z^{1}\right)^{\mu^{1}} \cdots\left(z^{L}\right)^{\mu^{L}}\right\rangle}{\left\langle 1-q^{g-2 k+2 m} t^{\beta}\left(z^{1}\right)^{\mu^{1}} \cdots\left(z^{L}\right)^{\mu^{L}}\right\rangle}\right)^{\frac{m n \vec{\mu}, g, \beta}{{ }^{3} \vec{\mu}}} .
\end{aligned}
$$

4.3. The case of the unknot. In Proposition 10.2 of [5], we have computed the free energy associated to the orthogonal Chern-Simons partition function of the knot as

$$
F^{S O}(\bigcirc ; q, t ; z)=\sum_{k=1}^{\infty} \frac{1}{k}\left(1+\frac{t^{k}-t^{-k}}{q^{k}-q^{-k}}\right) p b_{k}(z) .
$$

Thus we have

$$
\begin{aligned}
& F^{S O}(\bigcirc ; q, t ; w)-F^{S O}(\bigcirc ; q,-t ; w) \\
& =\sum_{k \in O \mathbb{Z}_{+}} \frac{2}{k} \frac{t^{k}-t^{-k}}{q^{k}-q^{-k}} p b_{k}(w) \\
& =\sum_{k \in O \mathbb{Z}_{+}} \frac{2}{k} \frac{t^{k}-t^{-k}}{[k]^{2}} p b_{k}(z) .
\end{aligned}
$$

Compared with (4.7), we obtain

$$
N_{(1), 0,1}=-N_{(1), 0,-1}=1 .
$$


All other coefficients $N_{B ; g, Q}$ are zero.

Thus we have

$$
n_{\mu, g, \beta}=\delta_{\mu,(1)} \delta_{g, 0} \operatorname{sign}(\beta)
$$

and

$$
\frac{Z_{C S}^{S O}(\bigcirc ; q, t ; w)}{Z_{C S}^{S O}(\bigcirc ; q,-t ; w)}=\prod_{m=1}^{\infty} \prod_{i=-\infty}^{\infty}\left(\frac{\left(1+q^{2 m} t z_{i}\right)\left(1-q^{2 m} t^{-1} z_{i}\right)}{\left(1-q^{2 m} t z_{i}\right)\left(1+q^{2 m} t^{-1} z_{i}\right)}\right)^{m} .
$$

4.4. Symmetry property of $q \rightarrow q^{-1}$ in infinite product structure. In this subsection, we discuss a basic symmetric property of this infinite product structure obtained from the orthogonal LMOV partition function. Here we focus on the knot case only, while the case of links exactly follows from the same analysis. In the derivation of the infinite product formula, we assume $|q|<1$ for the Taylor expansion of $\frac{1}{[d]^{2}}$. In the case of $|q|>1$, the Taylor expansion is given by

$$
\frac{1}{[d]^{2}}=\sum_{m=1}^{\infty} m q^{-2 m d}
$$

Therefore, the infinite product formula will be read as

$$
\begin{aligned}
& \frac{Z_{C S}^{S O}(\mathcal{K} ; q, t ; w)}{Z_{C S}^{S O}(\mathcal{K} ; q,-t ; w)} \\
& =\prod_{\mu \neq 0} \prod_{g=0}^{\infty} \prod_{\beta \in \mathbb{Z}} \prod_{m=1}^{\infty} \prod_{k=0}^{g}\left(\frac{\left\langle 1+q^{g-2 k-2 m} t^{\beta} z^{\mu}\right\rangle}{\left\langle 1-q^{g-2 k-2 m} t^{\beta} z^{\mu}\right\rangle}\right)^{\frac{m_{n} \mu, g, \beta}{{ }^{\prime} \mu}} \\
& =\prod_{\mu \neq 0} \prod_{g=0}^{\infty} \prod_{\beta \in \mathbb{Z}} \prod_{m=1}^{\infty} \prod_{k=0}^{g}\left(\frac{\left\langle 1+q^{-g+2(g-k)-2 m} t^{\beta} z^{\mu}\right\rangle}{\left\langle 1-q^{-g+2(g-k)-2 m} t^{\beta} z^{\mu}\right\rangle}\right)^{\frac{m_{n}, g, \beta}{{ }^{\prime} \mu}} \\
& =\prod_{\mu \neq 0} \prod_{g=0}^{\infty} \prod_{\beta \in \mathbb{Z} m=1}^{\infty} \prod_{k=0}^{g}\left(\frac{\left\langle 1+q^{-g+2 k-2 m} t^{\beta} z^{\mu}\right\rangle}{\left\langle 1-q^{-g+2 k-2 m} t^{\beta} z^{\mu}\right\rangle}\right)^{\frac{m_{\mu, g, \beta}}{\mathfrak{s} \mu}} \\
& =\prod_{\mu \neq 0} \prod_{g=0}^{\infty} \prod_{\beta \in \mathbb{Z} m=1}^{\infty} \prod_{k=0}^{g}\left(\frac{\left\langle 1+\left(q^{-1}\right)^{g-2 k+2 m} t^{\beta} z^{\mu}\right\rangle}{\left\langle 1-\left(q^{-1}\right)^{g-2 k+2 m} t^{\beta} z^{\mu}\right\rangle}\right)^{\frac{m_{\mu, g, \beta}}{\jmath_{\mu}}} .
\end{aligned}
$$

This is the symmetry of $q \rightarrow q^{-1}$ for the infinite product formula.

\section{ACKNOWLEDGMENTS}

We thank Pan Peng for many valuable discussions and Shengmao Zhu for giving many helpful suggestions and for proofreading the paper.

\section{REFERENCES}

[1] Anna Beliakova and Christian Blanchet, Skein construction of idempotents in Birman-Murakami-Wenzl algebras, Math. Ann. 321 (2001), no. 2, 347-373, DOI 10.1007/s002080100233. MR:1866492 (2002h:57018)

[2] Vincent Bouchard, Bogdan Florea, and Marcos Mariño, Topological open string amplitudes on orientifolds, J. High Energy Phys. 2 (2005), 002, 35 pp. (electronic), DOI 10.1088/11266708/2005/02/002. MR 2140507 (2006h:81260)

[3] R. Brauer, On algebras which are connected with the semisimple continuous groups, Ann. Math. 63 (1937) 854-872. 
[4] Lin Chen, Chern-Simons theory of knots invariants, ProQuest LLC, Ann Arbor, MI, 2009. Thesis (Ph.D.)-University of California, Los Angeles. MR 2713712

[5] Lin Chen and Qingtao Chen, Orthogonal quantum group invariants of links, Pacific J. Math. 257 (2012), no. 2, 267-318, DOI 10.2140/pjm.2012.257.267. MR2972467

[6] Qingtao Chen, Some mathematical aspects of quantum field theory, ProQuest LLC, Ann Arbor, MI, 2009. Thesis (Ph.D.)-University of California, Berkeley. MR2717855

[7] P. Freyd, D. Yetter, J. Hoste, W. B. R. Lickorish, K. Millett, and A. Ocneanu, A new polynomial invariant of knots and links, Bull. Amer. Math. Soc. (N.S.) 12 (1985), no. 2, 239-246, DOI 10.1090/S0273-0979-1985-15361-3. MR776477 (86e:57007)

[8] Rajesh Gopakumar and Cumrun Vafa, On the gauge theory/geometry correspondence, Adv. Theor. Math. Phys. 3 (1999), no. 5, 1415-1443. MR.1796682 (2001k:81272)

[9] V. F. R. Jones, A new knot polynomial and von Neumann algebras, Notices Amer. Math. Soc. 33 (1986), no. 2, 219-225. MR830613(87d:57007)

[10] V. F. R. Jones, Hecke algebra representations of braid groups and link polynomials, Ann. of Math. (2) 126 (1987), no. 2, 335-388, DOI 10.2307/1971403. MR.908150 (89c:46092)

[11] Louis H. Kauffman, State models and the Jones polynomial, Topology 26 (1987), no. 3, 395-407, DOI 10.1016/0040-9383(87)90009-7. MR899057 (88f:57006)

[12] Louis H. Kauffman, An invariant of regular isotopy, Trans. Amer. Math. Soc. 318 (1990), no. 2, 417-471, DOI 10.2307/2001315. MR.958895 (90g:57007)

[13] José M. F. Labastida and Marcos Mariño, A new point of view in the theory of knot and link invariants, J. Knot Theory Ramifications 11 (2002), no. 2, 173-197, DOI 10.1142/S0218216502001561. MR1895369(2003h:57016)

[14] José M. F. Labastida, Marcos Mariño, and Cumrun Vafa, Knots, links and branes at large N, J. High Energy Phys. 11 (2000), Paper 7, 42, DOI 10.1088/1126-6708/2000/11/007. MR:1806596(2003b:57015)

[15] W. B. R. Lickorish and Kenneth C. Millett, A polynomial invariant of oriented links, Topology 26 (1987), no. 1, 107-141, DOI 10.1016/0040-9383(87)90025-5. MR880512(88b:57012)

[16] Chiu-Chu Melissa Liu, Kefeng Liu, and Jian Zhou, A proof of a conjecture of Mariño-Vafa on Hodge integrals, J. Differential Geom. 65 (2003), no. 2, 289-340. MR2058264(2005m:14104)

[17] Kefeng Liu and Pan Peng, On a proof of the Labastida-Mariño-Ooguri-Vafa conjecture, Math. Res. Lett. 17 (2010), no. 3, 493-506, DOI 10.4310/MRL.2010.v17.n3.a9. MR2653684 (2011d:58059)

[18] Kefeng Liu and Pan Peng, Proof of the Labastida-Mariño-Ooguri-Vafa conjecture, J. Differential Geom. 85 (2010), no. 3, 479-525. MR2739811 (2012a:57019)

[19] Kefeng Liu and Pan Peng, New structures of knot invariants, Commun. Number Theory Phys. 5 (2011), no. 3, 601-615, DOI 10.4310/CNTP.2011.v5.n3.a1. MR2864461

[20] Marcos Mariño, String theory and the Kauffman polynomial, Comm. Math. Phys. 298 (2010), no. 3, 613-643, DOI 10.1007/s00220-010-1088-6. MR2670922 (2011g:57016)

[21] Hirosi Ooguri and Cumrun Vafa, Knot invariants and topological strings, Nuclear Phys. B 577 (2000), no. 3, 419-438, DOI 10.1016/S0550-3213(00)00118-8. MR.1765411(2001i:81254)

[22] Józef H. Przytycki and Paweł Traczyk, Invariants of links of Conway type, Kobe J. Math. 4 (1988), no. 2, 115-139. MR945888 (89h:57006)

[23] Arun Ram, A Frobenius formula for the characters of the Hecke algebras, Invent. Math. 106 (1991), no. 3, 461-488, DOI 10.1007/BF01243921. MR1134480 (93c:20029)

[24] Arun Ram, Characters of Brauer's centralizer algebras, Pacific J. Math. 169 (1995), no. 1, 173-200. MR 1346252 (96k:20020)

[25] Arun Ram, A "second orthogonality relation" for characters of Brauer algebras, European J. Combin. 18 (1997), no. 6, 685-706, DOI 10.1006/eujc.1996.0132. MR1468338 (98m:20015)

[26] N. Reshetikhin and V. G. Turaev, Invariants of 3-manifolds via link polynomials and quantum groups, Invent. Math. 103 (1991), no. 3, 547-597, DOI 10.1007/BF01239527. MR1091619 (92b:57024)

[27] N. Yu. Reshetikhin and V. G. Turaev, Ribbon graphs and their invariants derived from quantum groups, Comm. Math. Phys. 127 (1990), no. 1, 1-26. MR1036112 (91c:57016)

[28] Hans Wenzl, On the structure of Brauer's centralizer algebras, Ann. of Math. (2) 128 (1988), no. 1, 173-193, DOI 10.2307/1971466. MR.951511 (89h:20059) 
[29] Edward Witten, Quantum field theory and the Jones polynomial, Comm. Math. Phys. 121 (1989), no. 3, 351-399. MR990772 (90h:57009)

Mathematics Section, International Center for Theoretical Physics, Strada Costiera, 11, Trieste, I-34151, Italy

E-mail address: qchen1@ictp.it

Center of Mathematical Sciences, Zhejiang University, Box 310027, Hangzhou, PeoPle's Republic of China - and - Department of Mathematics, University of California At Los Angeles, Box 951555, Los Angeles, California 90095-1555

E-mail address: liu@math.ucla.edu 